\title{
Single-Mode and Polarization-Independent Silicon-on-Insulator Waveguides With Small Cross Section
}

\author{
Seong Phun Chan, Ching Eng Png, Soon Thor Lim, Graham T. Reed, and Vittorio M. N. Passaro, Member, IEEE
}

\begin{abstract}
The fabrication restrictions that must be imposed on the geometry of optical waveguides to make them behave as single-mode devices are well known for relatively large waveguides, with shallow etch depth. However, the restrictions for small waveguides ( $\sim 1 \mu \mathrm{m}$ or less in cross section) are not well understood. Furthermore, it is usually a requirement that these waveguides are polarization independent, which further complicates the issues. This paper reports on the simulations of the conditions for both single-mode behavior and polarization independence, for small and deeply etched silicon-on-insulator (SOI) waveguides. The aim is to satisfy both conditions simultaneously. The results show that at larger waveguide widths, waveguide etch depth has little effect on the mode birefringence because the transverse-electric (TE) mode (horizontal-polarized mode) is well confined under the rib region. However, at smaller rib widths, the etch depth has a large influence on birefringence. An approximate equation relating the rib-waveguide width and etch depth to obtain polarization-independent operation is derived. It is possible to achieve single-mode operation at both polarizations while maintaining polarization independence for each of the waveguide heights used in this paper but may be difficult for other dimensions. For example, a 1- $\mu \mathrm{m}$ SOI rib waveguide with an etch depth of $0.64 \mu \mathrm{m}$ and rib width of $0.52 \mu \mathrm{m}$ is predicted to exhibit such characteristics.
\end{abstract}

Index Terms-Polarization independence, rib waveguides, silicon-on-insulator (SOI), single-mode condition (SMC).

\section{INTRODUCTION}

$\mathbf{S}$ INCE the early investigation of propagation loss and polarization dependence of silicon-on-insulator (SOI) waveguides, the interest in SOI photonic devices has increased enormously. Research activities in SOI range from the study of basic building blocks such as waveguides [5]-[9] and bends [14] to more complex devices such as modulators [1], ring resonators [2], Bragg gratings [3], and even light sources in silicon [4]. Single-mode SOI rib waveguides with a large cross section have been studied extensively by a number of researchers [5]-[9] to

Manuscript received December 10, 2004; revised March 19, 2005. The work of S. P. Chan and S. T. Lim is supported in part by Agilent Technologies, Singapore, and the University of Surrey, Surrey, U.K. The work of C. E. Png is supported in part by the Overseas Research Student (ORS) Award Scheme, Bookham Technology, Inc., and the Institution of Electrical Engineers (IEE).

S. P. Chan, S. T. Lim, and G. T. Reed are with the Advanced Technology Institute, School of Electronics and Physical Sciences, University of Surrey, Guildford, Surrey GU2 7XH, U.K. (e-mail: s.chan@surrey.ac.uk; eep1s1@surrey.ac.uk; g.reed@surrey.ac.uk).

C. E. Png is with the Institute of High Performance Computing, A*Star, Singapore Science Park II, Singapore 117528 Singapore (e-mail: pngce@ihpc. a-star.edu.sg).

V. M. N. Passaro is with the Dipartimento di Elettrotecnica ed Elettronica, Politecnico di Bari, 70125 Bari, Italy (e-mail: passaro@dee-mail.poliba.it).

Digital Object Identifier 10.1109/JLT.2005.849883 find single-mode behavior at the same time as low propagation loss. The majority of these photonic devices in SOI have been studied in waveguides that are multimicrometers in cross-sectional dimensions (of the order of $5 \mu \mathrm{m}$ ) to facilitate low-loss coupling to and from optical fibers. Soref et al. [5] first proposed a simple expression for these large ribs waveguides, related to their geometry to ensure that they satisfied the single-mode condition (SMC), as follows:

$$
\begin{aligned}
& \frac{W}{H} \leq \alpha+\frac{r}{\sqrt{1-r^{2}}} \\
& \text { for } 0.5 \leq r \leq 1.0
\end{aligned}
$$

where $r$ is the ratio of slab height to overall rib height, $W / H$ is the ratio of waveguide width to overall rib height, and $\alpha=0.3$. The analysis of the waveguides was limited to shallow-etched ribs as stated in (2) $(r<0.5)$, where deeply etched rib waveguides are not considered. Furthermore, the waveguide dimensions are larger than the operating wavelength. Their analysis was based on the assumption that high-order vertical modes (i.e., modes other than the fundamental mode) confined under the rib waveguides were coupled to the outer slab region during propagation, therefore yielding high propagation losses for the higher order modes. Thus, the waveguides behave as single-mode waveguides, as all other modes are lost. The analytical effective-index method (EIM) was used by Pogossian $e t$ al. [6] for a similar analysis and suggested that more stringent geometrical constraints needed to be imposed for single-mode operation, resulting in an alternative value of $\alpha=0$ in (1). Powell [7] also made a more general analysis for rib waveguides with vertical or angled sidewalls, finding a simple analytical expression for such waveguides that was in good agreement with the original work of Soref et al. [5], as well as using a simple scalar approximation in his analysis. He showed that the scalar approximation is sufficiently accurate for large rib waveguides with shallow etch depth (large $r$ ), where the mode profiles are similar for both polarizations.

The widely used design criteria stated in (1) was revisited recently [9] using the full-vectorial beam-propagation method (BPM) to demonstrate this simple equation is not sufficient to ensure single-mode behavior. The prediction by the design formula (1) that waveguides should be single-mode was questioned in this analysis when some waveguides were shown to support other higher order vertical modes. Nevertheless, this and all the other work presented on this specific issue to date has been limited to waveguides of large cross section with shallow etch depths $(r<0.5)$. 


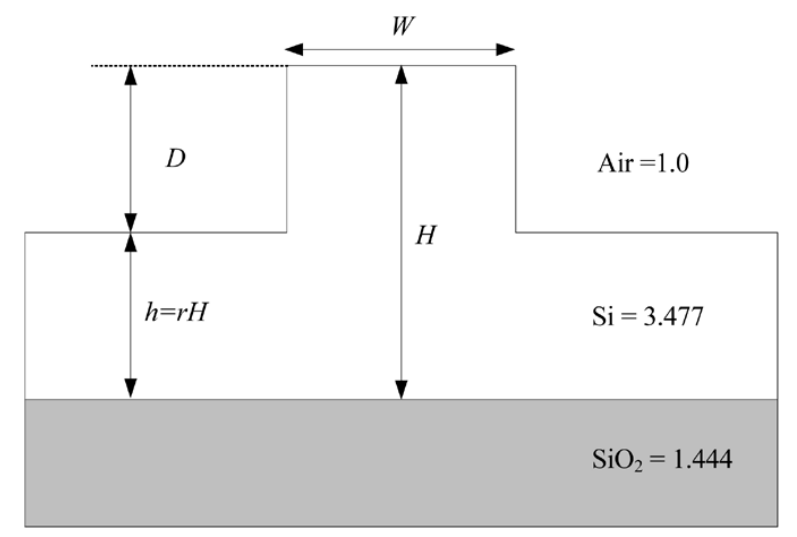

Fig. 1. Cross section and waveguide parameters of an SOI rib waveguide.

However, there is a current trend in silicon photonic circuits to move to smaller device dimensions for improved cost efficiency and device performance. The trend can also come at some cost to performance, notably in the polarization dependence of the circuits if they are not carefully designed. Furthermore, the validity of the design equation (1), applied to small and deeply etched rib waveguides, has not been sufficiently addressed. The objective of this paper is to discuss the polarization dependence and single-mode conditions for smaller waveguide dimensions with deeply etched ribs. The degree of influence of etch depth on waveguide performance also becomes apparent when small waveguides are designed for polarization independence. The issues associated with work on the polarization independence and single-mode conditions for smaller waveguide dimensions is discussed and examples of designs that satisfy both requirements are given. In order to maintain the consistency with other work [5], [7]-[9], the full-vectorial BPM was used to analyze the waveguide structure in Fig. 1 for single-mode propagation and polarization independence. Some results of the SMC condition have also been verified by the finite-element method (FEM) [11].

\section{SingLe-Mode CONDITION FOR SMALL-CROSS-SECTION WAVEGUIDES}

The modal analysis of the small-cross-section SOI rib waveguides is based on the BPM algorithm [10] using the correlation mode-solving technique, particularly useful for $z$-invariant structures. In the correlation approach, an arbitrary field is launched for each dominant polarization into the waveguide structure off-center, to excite all the possible modes and allow propagation in the $z$ direction via normal BPM. During the propagation stage, the following correlation function between the input field and the propagating field is computed:

$$
P(z)=\iint \phi_{\text {input }}(x, y, 0) \phi(x, y, z) d x d y .
$$

The correlation method resolves all high-order modes supported in the structure by determining the mode spectrum, where eigenvalues or the effective-index value of all guided modes and leaky modes are found. The computed mode spectrum is the fast Fourier transform (FFT) of the correlation function. It will exhibit a peak at the frequency of each of the guided mode, with a height equal to the fraction of power in the launched field contained in the mode. To maintain consistency of the analysis with others, we have employed the notation [5], [9] of $E H_{\mathrm{nm}}$ and $H E_{\mathrm{nm}}$, where $n=0,1,2,3 \ldots$, $m=0,1,2,3 \ldots$, and where $E H$ refers to the dominant vertical polarization and $H E$ to horizontal polarization. They are commonly known as quasi-TE modes for $H E_{\mathrm{nm}}$ modes and quasi-transverse magnetic (quasi-TM) for $E H_{\mathrm{nm}}$ modes.

The single-mode simulation was set up using the waveguide structure shown in Fig. 1, which is a rib waveguide of silicon $\left(n_{g}=3.477\right)$ on silica $\left(n_{s}=1.444\right)$ and has an upper cladding that is air $\left(n_{c}=1\right)$. SOI rib waveguides with an overall height of $1.00,1.35$, and $1.50 \mu \mathrm{m}$ are analyzed at a wavelength of $1.55 \mu \mathrm{m}$, related to examples of design geometries used in our previous papers, such as silicon optical modulators [1], ring resonators [2], and waveguide-based Bragg gratings [3]. We have made the assumption of no coupling between the quasi-TE and quasi-TM modes. The energy fields in both majority and minority components are taken into consideration because a minority field component has the potential of changing the effective-index value for small waveguide cross sections. A Gaussian field was chosen to approximate an optical fiber mode, and it is deliberately launched off-center at one third of the waveguide width to excite high-order modes supported in the structure. The propagation length was chosen to be $4 \mathrm{~mm}$, which is sufficiently long to allow potentially guided higher order modes to leak out from the rib waveguide. A number of vertical and horizontal guided modes is restricted to three $\left(H E_{n 0}, n<3\right.$, and $E H_{0 m}, m<3$ ) for both polarizations during the computation, in order to minimize the computation time. However, this is more than sufficient to determine the single-mode-multimode transition. In the simulations, the waveguide height $(H)$, etch depth $(D)$, and slab height $(h)$ were kept constant while increasing the waveguide width in increments of $0.01 \mu \mathrm{m}$ to find the boundary between single-mode and multimode behavior. The iteration of the simulation is repeated with different values of the slab height $(h)$ to waveguide height $(H)$ ratio (parameter $r=h / H)$. Hence, the process is one in which we gradually increase the waveguide width until a second-order mode is guided $\left(E H_{02}, H E_{02}\right)$. The boundary of the single mode/multimode can therefore be determined by computing the minimum waveguide width at which the first higher order mode is supported by the waveguide structure.

In Fig. 2, our simulation results are presented together with a scalar approximation [7] and other approaches of Soref et al. [5] and Pogossian et al. [6], for an SOI rib waveguide height of $1.50 \mu \mathrm{m}$ and operating wavelength $\lambda=1550 \mathrm{~nm}$. It should be noted that the original data of [5] and [6] does not extend to the range of $r<0.5$, and so here we have simply extended these results by extrapolation. Fig. 2 shows that the scalar approximation and quasi-TE $(H E)$ results are very similar for a large range of values of parameter $r$ (above $\sim r=0.4$ ). This scalar approximation result also approaches the results of [6] for values of parameter $r$ less than approximately 0.40. However, it can be seen that the results of the quasi-TM $(E H)$ that simulation is very different from both the scalar and quasi-TE computation for $r<0.5$, where the boundary of the single mode/multimode regime for $E H$ and $H E$ modes diverge. This simulation data suggests that the single-mode cutoff condition 


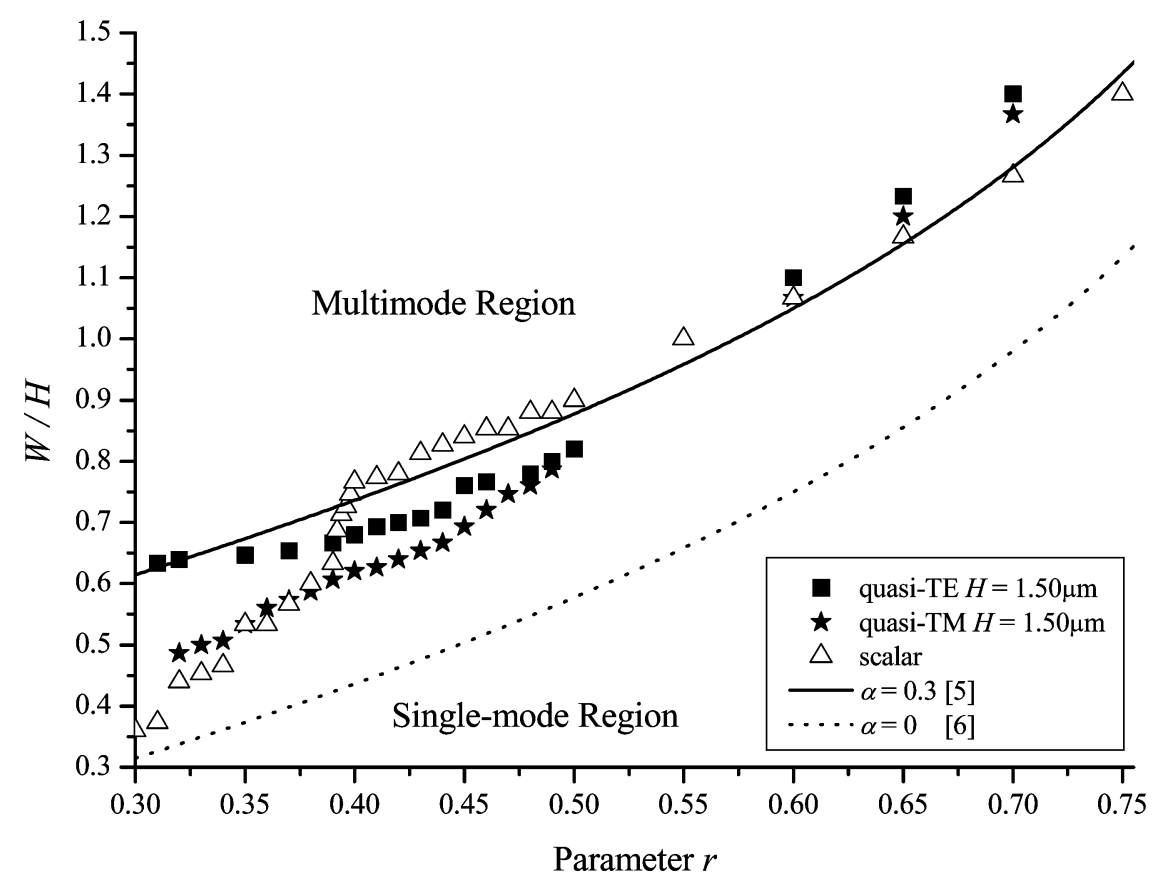

Fig. 2. Comparison of scalar and full-vectorial numerical simulation methods for quasi-TE and quasi-TM single-mode cutoff dimensions for a rib waveguide height of $1.50 \mu \mathrm{m}$. The trend lines in the plot indicate the boundary between single-mode and multimode regions.

for deeply etched rib waveguides is different from commonly used design rules [5], [6] and the strict criteria for the cutoff condition, where parameter $\alpha=0$ [6], are less useful than the other simulations, either for small or large values of parameter $r$. This also reaffirms that the work of Soref $e t$ al. [1], or even that of the scalar approximation, can be used as a basis of design of single-mode waveguides for shallow-etched rib waveguides $(r>0.5)$ with a large cross section. The primary issues, however, are the differences between the SMCs for different polarizations at smaller values of parameter $r$, which are significant, particularly in small waveguides. This is because the optical confinement for such small waveguides is dominated by the boundary conditions, which are well known to be different for quasi-TE and quasi-TM modes.

Our initial investigation for waveguide height $H=1.50 \mu \mathrm{m}$ was presented in conventional notation for a rib-waveguide geometry depicted in Fig. 1. It is instructive, however, to plot the data associated with parameter $r$ in a different way, which is related to waveguide etch depth $(D)$. In the remainder of this paper, we adopt this approach as it clarifies some of the design issues. If we turn our attention to the design of birefringence-free waveguides, we can also provide guidelines for achieving both single-mode behavior and birefringence free behavior simultaneously.

\section{POLARIZATION INDEPENDENCE}

The increased polarization dependence in small waveguides is derived from the increasingly differing mode shapes of the TE and TM modes. The question arises as to whether the prime concern is to maintain similar losses for the TE and TM modes or to provide similar propagation constants in order to maintain similar phase performance for interferometric-based devices, because it is not generally possible to maintain both. In principle, waveguide birefringence can be removed by appropriate choice of rib-waveguide width-to-height ratio [3], [8] or by employing stress engineering [12] to tailor the thickness of $\mathrm{SiO}_{2}$ for polarization compensation. Similarly, as discussed previously, it is also possible to design a rib waveguide supporting only a single mode with an appropriate selection of the rib width and height [8], [9]. The degree of influence of waveguide width and etch depth on waveguide birefringence for a given rib height is demonstrated by further simulations here. Once again, we have used rib-waveguide heights of 1.00 , 1.35 , and $1.50 \mu \mathrm{m}$, and have evaluated the effective indexes of the fundamental quasi-TE and quasi-TM modes. If we assume an operating wavelength of $1550 \mathrm{~nm}$, we can produce a graph of the variation of the TE/TM fundamental mode effective-index difference $\left(\Delta N=N_{\mathrm{TE}}-N_{\mathrm{TM}}\right)$ for various etch depths $(D)$ and waveguide widths $(W)$.

Fig. 3(a)-(c) shows various curves of waveguide width against the effective-index difference, each for a different waveguide height. By using an appropriate etch depth for each waveguide height, the curves cross the zero-birefringence (ZBR) axis twice when the effective indexes of both polarization modes is the same, which indicates it is possible to produce birefringence-free waveguides of two different waveguide widths when a deep etch depth is employed $(r<0.5)$. The wider waveguide width is highly preferable compared with its narrower counterpart when we consider that the wider devices will result in more flexibility during fabrication, such as the accuracy of the etch depth. If we consider $H=1.50 \mu \mathrm{m}$, $D=0.90 \mu \mathrm{m}$, the birefringence-free waveguide widths $W$ can be determined from Fig. 3(c) as 0.90 and $1.05 \mu \mathrm{m}$. If we now introduce etch-depth uncertainties of $10 \mathrm{~nm}$, we see that the latter width is more desirable as the impact of the uncertainty on change in birefringence is reduced from $6.03 \times 10^{-4}$ to 


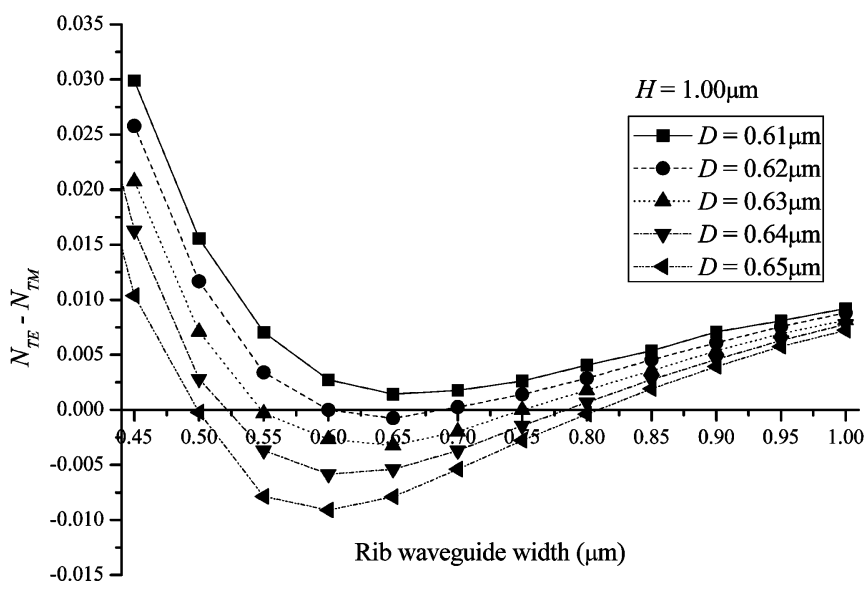

(a)

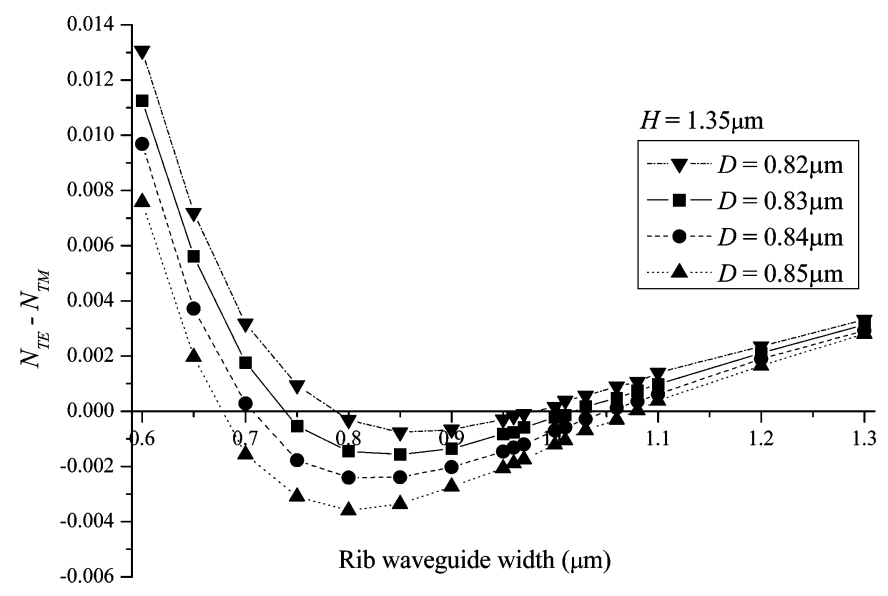

(b)

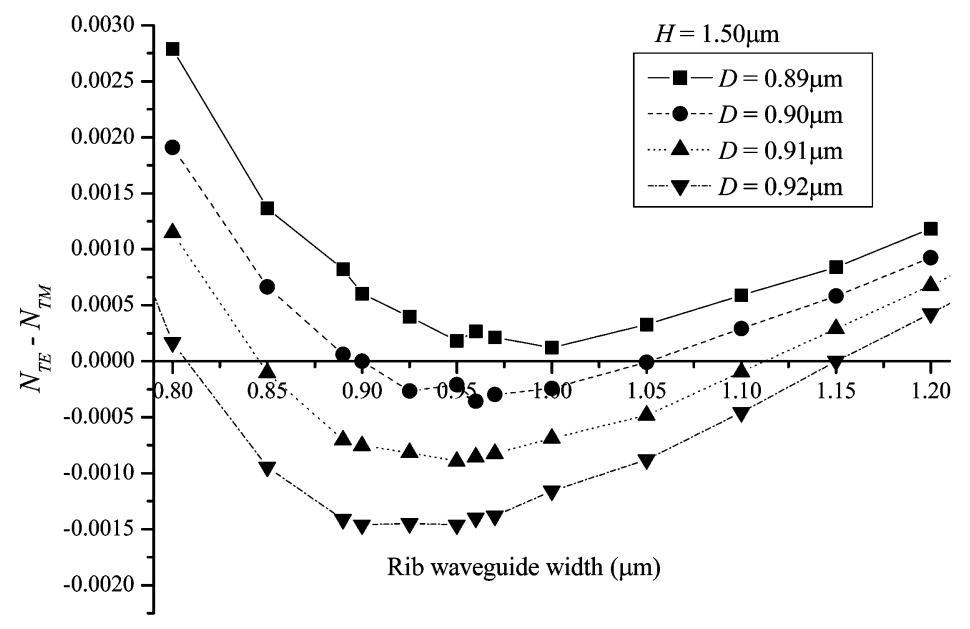

(c)

Fig. 3. Effective-index difference calculation between quasi-TE and quasi-TM polarized modes using the FEM [11], for waveguide heights (a) $1.00 \mu \mathrm{m}$, (b) $1.35 \mu \mathrm{m}$, and (c) $1.50 \mu \mathrm{m}$.

$3.28 \times 10^{-4}$. The same trend can also be observed in Fig. 3(a) and (b). The study on etch control of silicon in $0.13-\mu \mathrm{m}$ process technology has determined experimentally [16] that the etch-depth tolerances are of the order of $\pm 1 \%$ to $\pm 2 \%$. Therefore, if we consider a $1-\mu \mathrm{m}$ etch depth to form an SOI waveguiding structure, the tolerance will be of the order of $\pm 10 \mathrm{~nm}$. As process technology moves to the next generation beyond the critical dimensions (CDs) of $90 \mathrm{~nm}$, following the International Technology Roadmap for Semiconductors (ITRS) [17], the reactive ion etching (RIE) process control will be improved and continue to be driven by the $\mathrm{CD}$ requirement of advanced lithographic processes. However, most optical devices made from silicon are likely to have CDs of hundreds of nanometers for the foreseeable future, which means that the infrastructure already exists for the next several generations of optical circuits, if they are fabricated in silicon.

A gradual increase of the etch depth is also used in the simulations to show the influence of etch depth on the modal birefringence. The result shows that at larger waveguide widths, waveguide etch depth has little effect on the mode birefringence because the TE mode (horizontal polarized mode) is well confined under the rib region. However, at smaller rib widths, the etch depth has a large influence on birefringence. It is clear that in each of the plots in Fig. 3, the curves have gone through three transitions. For large waveguide widths, the TE-mode effective-index dominates as most power is confined under the rib region; this makes the device exhibit slablike behavior, allowing higher order modes to couple to the slab region. As the width is reduced gradually, the effective index of the TM mode becomes similar to that of the TE mode, and then the effective-index difference can become negative. When the waveguide width becomes very small, most of the mode power will again be confined under the slab regions, and as a result, the TE effective indexes once again become higher than those of the TM modes. To confirm the trends of the data in the simulations, some of data in Fig. 3 have been computed by the full-vectorial FEM [11], finding good agreement with results produced by BPM.

Drawbacks associated with small waveguide geometries are that it is difficult to fabricate such devices accurately, and it becomes difficult to couple light efficiently into the waveguides. Although computer simulations enable us to generate detailed designs of rib waveguides with polarization independence, we must take into account variations in the fabrication process such as etch depth uniformity, etching profile, and $\mathrm{CD}$, as well as process control of waveguide width. Real-time in situ monitoring of microelectromechanical systems (MEMS) using deep RIE processing has been demonstrated [18] in high-aspect-ratio etching in SOI wafers. Process variables in RIE also 


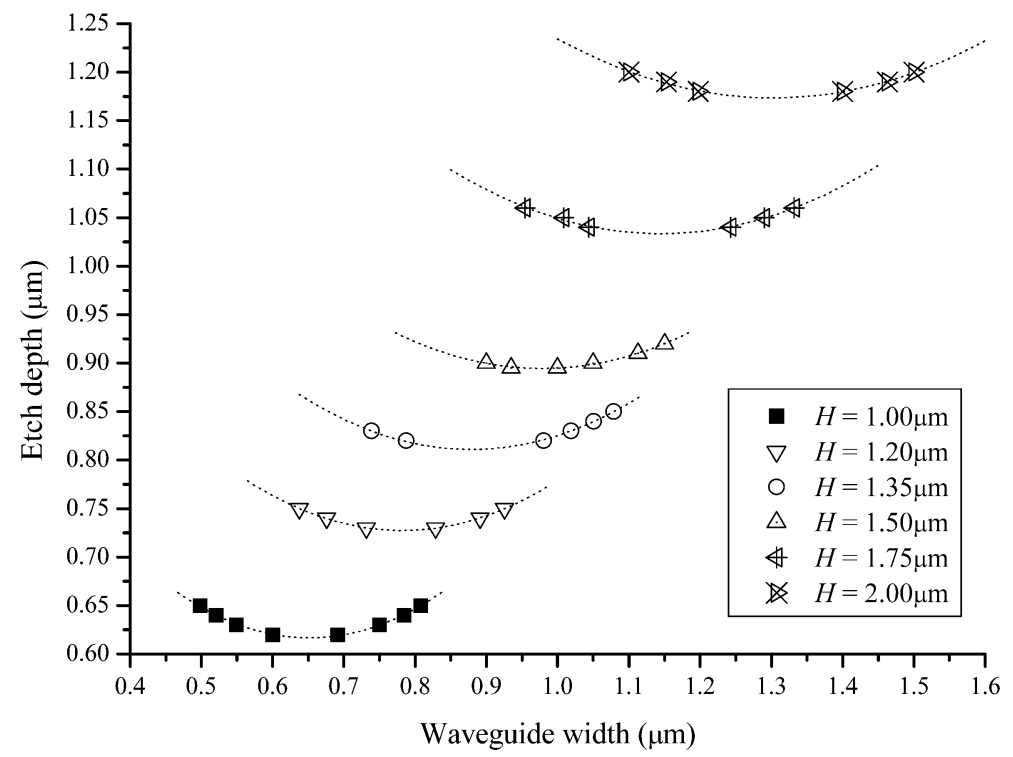

Fig. 4. Etch-depth influence on waveguide width in SOI structure to support ZBR for various waveguide heights at $\lambda=1550 \mathrm{~nm}$, reflecting the etch-depth tolerance requirements in relation to process technology.

have been studied extensively in [19]. Both approaches are nondestructive and provide real-time process analysis. Hence, this will lead to improved process accuracy and controllable process repeatability.

If we now plot the locus of the points in Fig. 3 that cross the ZBR axis, we observe similar trends for all three rib waveguide heights. We have also added simulated ZBR data for waveguide heights of $H=1.20,1.75$, and $2.00 \mu \mathrm{m}$. This is shown in Fig. 4, where we plot etch depth against waveguide width for a variety of waveguide heights and fit with the second-order polynomial. We note that there is a minimum etch depth $\left(D_{\min }\right)$ for each waveguide design, which must be matched to a specific waveguide width if the polarization-independent condition is to be met. Hence, we can use this data to predict the rib waveguide width and etch depth needed for polarization-independent operation by assuming that the relationship between $D_{\min }$ and waveguide height is linear. This relationship can be expressed by the following approximate equation after linear regression fitting of simulation data, for waveguide heights $H<2 \mu \mathrm{m}$ :

$$
D_{\min }=0.06 \times 10^{-6}+0.556 H \text {. }
$$

This finding is particularly useful to aid the design of waveguides meeting the requirement of polarization independence. The low curve gradient at the minimum etch depth depicted in Fig. 4 also makes the minimum etch depth a good choice from a fabrication perspective, because the impact of the tolerances of the etching process is minimized. It is also interesting to note that as a proportion of the waveguide height $\left(D_{\min } / H\right)$, the minimum etch depth increases as the waveguide height is reduced, which in turn means that for smaller waveguide height, the smaller the maximum value of parameter $r$ becomes and a higher etching tolerance must be satisfied to maintain the ZBR condition. For instance, an etch depth error of $10 \mathrm{~nm}$ will introduce a modal birefringence difference of $3.2 \times 10^{-4}$ for

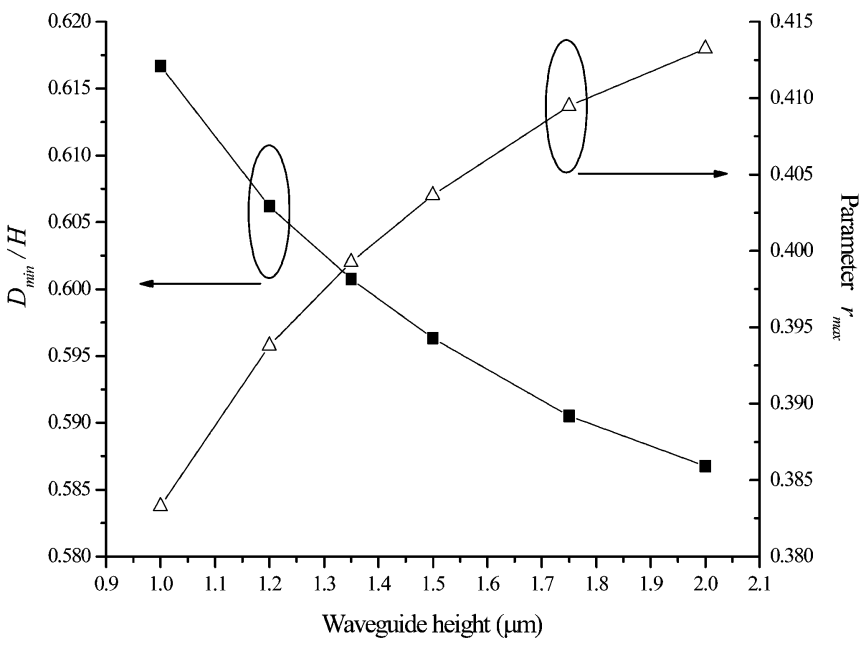

Fig. 5. Minimum etch depth to waveguide height $\left(D_{\min } / H\right)$ ratio and parameter $r$ plotted as a function of waveguide height. This relationship indicates the requirement of minimum etch-depth tolerance to achieve ZBR increases as the waveguide height is reduced.

$H=1.50 \mu \mathrm{m}$ compared with $2.7 \times 10^{-3}$ for $H=1.00 \mu \mathrm{m}$. This is clearly illustrated in Fig. 5 and can be expressed as

$$
\begin{aligned}
& r_{\max }=1-\frac{D_{\min }}{H}=1-\frac{0.06 \times 10^{-6}+0.556 H}{H} \\
& r_{\max }=0.444-\frac{0.06 \times 10^{-6}}{H} .
\end{aligned}
$$

\section{SATISFYing Both ReQuiREMENTS SimultaneOUSLY}

Although the requirements for ZBR and single-mode operation can be met using appropriate rib design, so far in this paper, both requirements have not been presented simultaneously when small waveguide dimensions are considered. We have combined the SMC for quasi-TE and quasi-TM modes $(H=1.00,1.35$, and $1.50 \mu \mathrm{m})$ together with ZBR curves to 


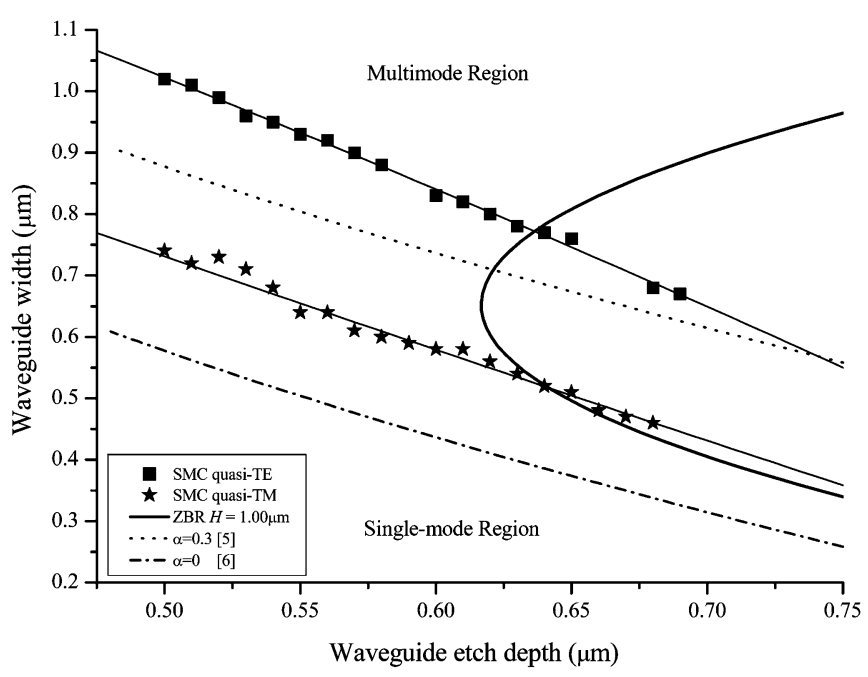

(a)

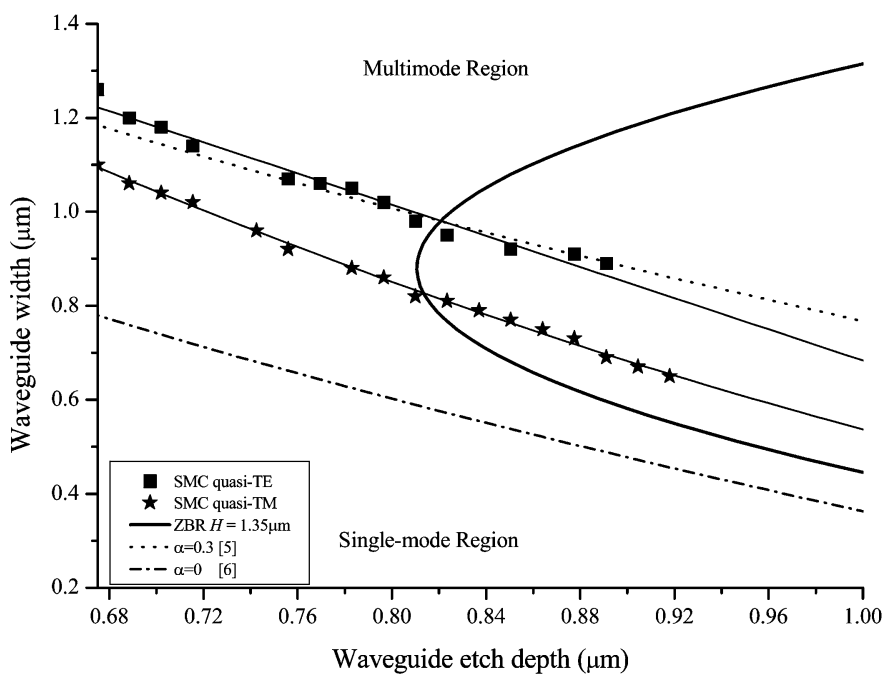

(b)

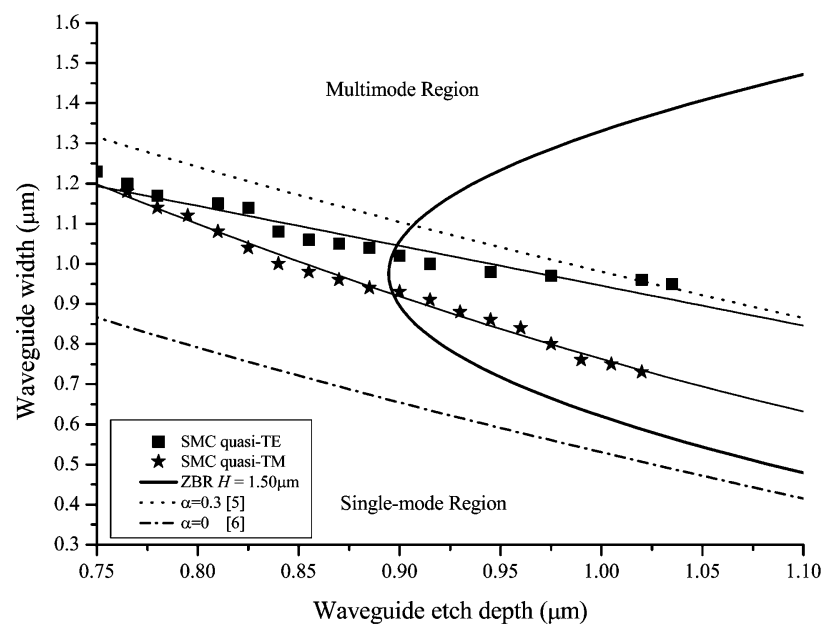

(c)

Fig. 6. Trend and boundary lines for single-mode cutoff dimensions and ZBR as a function of waveguide dimensions for an operating wavelength of $\lambda=1550 \mathrm{~nm}$ for (a) $H=1.00 \mu \mathrm{m}$, (b) $1.35 \mu \mathrm{m}$, and (c) $1.50 \mu \mathrm{m}$.

demonstrate that both conditions can be met under certain circumstances. Unlike other authors, we have presented the data in terms of the absolute waveguide dimensions rather than using normalized values of $W / H$ ratio and parameter $r$, because this provides a clearer indication of the design problem. All the data points that cross the ZBR axis in Fig. 3 were used in Fig. 4 in terms of waveguide width $(W)$ and etch depth $(D)$ to plot a locus of the birefringence-free condition for varying waveguide dimensions. This data is now used to show the relationship between single-mode operation and birefringence-free operation and the possibility of satisfying both conditions simultaneously. Simulation data of waveguide heights $H=1.0,1.35$, and $1.50 \mu \mathrm{m}$ are again used. Experimental results of [2], in which the device exhibits low polarization dependence and supports only a single mode, give us confidence in our numerical results. Fig. 6(a)-(c) shows plots of both the SMC and the birefringence-free locus for each of the three waveguide heights. These figures suggest that it is possible to achieve single-mode operation at both polarizations while maintaining polarization independence for each of the waveguide heights used. In order to satisfy both the requirements of single-mode operation and

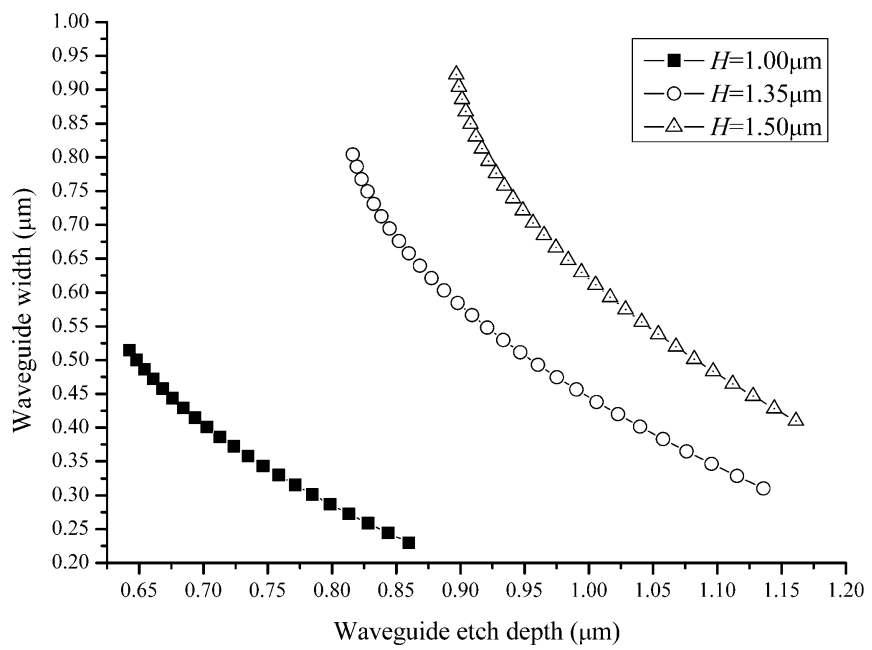

Fig. 7. Waveguide geometries that exhibit single-mode and birefringence-free behavior simultaneously for waveguide heights $H=1.00,1.35$, and $1.50 \mu \mathrm{m}$ at a design wavelength of $\lambda=1550 \mathrm{~nm}$.

polarization independence, we must choose a point on the birefringence-free locus that is below the single-mode boundary 


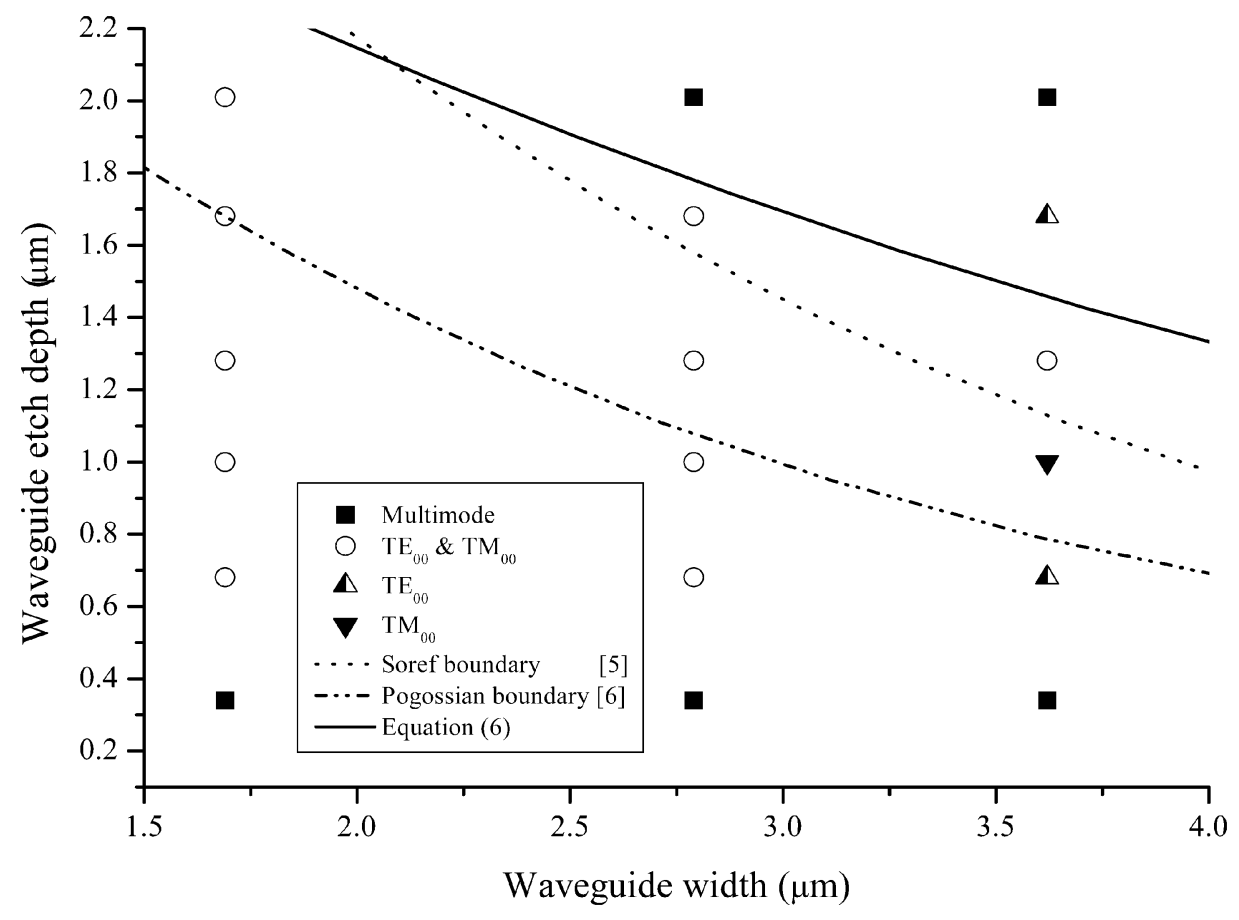

Fig. 8. Comparison of our design equation for a small-cross-section SOI waveguide with different design rules in the literature together with experimental observation for large-waveguide dimensions from [13].

for both quasi-TE and quasi-TM modes. However, it should be noted that the trend lines are approximate because they are the result of curve fitting, and therefore, for a specific design, data points should be used instead of the trend lines.

The design rules for the SMC $\alpha=0.3$ [5] and $\alpha=0$ [6] were also extrapolated for comparison with our numerical simulation. It is interesting to note that the intersection between the birefringence-free locus and the quasi-TE graphs determines the limit of the waveguide width to guide not only the fundamental TE mode, but multiple TM modes. For instance, the maximum values of rib width $(W)$ are $0.77,0.98$, and $1.05 \mu \mathrm{m}$ for waveguide heights $(H)$ of $1.00,1.35$, and $1.50 \mu \mathrm{m}$, respectively, to satisfy the quasi-TE SMC and the birefringence-free condition. However, if we also wish to satisfy the SMC for TM polarization, narrower waveguides are required. In this case the waveguide widths must be $0.52,0.83$, and $0.92 \mu \mathrm{m}$, respectively. The crossing points for the two polarizations between the birefringence-free condition and both single-mode boundary lines are closer to each other as the waveguide height is increased. Hence, it is easier to satisfy both conditions for waveguide heights of 1.35 and $1.50 \mu \mathrm{m}$ compared with waveguides with a height of $1.00 \mu \mathrm{m}$ or less in the context of fabrication tolerance. As the etch depth $(D)$ increases (or parameter $r$ decreases), the waveguide width must also be reduced to maintain only single-mode operation for a designated polarization. This can be observed for all the ZBR data points below which the quasi-TM boundary line intersects the ZBR locus plots in Fig. 7. These results of SMC and ZBR curves give us confidence in the possibility to achieve SOI waveguides satisfying both requirements, as required in many applications. However, in some cases, the relevant requirement of the waveguide width may be too narrow for fabrication purposes and impractical when coupling light into the small-waveguide structure. The SMC for these small waveguides with deep etch depth $(r<0.5)$ is effectively limited by the boundary condition set by the quasi-TM mode. We have produced a generalized equation in terms of waveguide dimensions, following the notation used in [5]

$$
\begin{aligned}
& \frac{W}{H} \leq 0.05+\frac{(0.94+0.25 H) r}{\sqrt{1-r^{2}}} \\
& 0.3<r<0.5 \text { and } 1.0 \leq H \leq 1.5 .
\end{aligned}
$$

It is interesting to examine the usefulness of the above approximate equation by comparing with experimental SOI waveguide devices. Following the approach given in [6], we have compared the experimental results from Rickman et al. [13] who experimentally investigated the dependency of waveguide geometries supporting only fundamental-mode operation, to our approximate equation, even though the results of [6] are based upon waveguides much larger than those simulated in this paper. We have adopted this approach because there is insufficient experimental data in the literature to compare and study with our modeling, to unequivocally confirm our design rules. However, our simulation results were in reasonable agreement with the experimental observations for waveguide height of $4.3 \mu \mathrm{m}$, even for large etch depths [13]. We can observe from Fig. 8 that, in contrast to the boundary prediction of both Pogossian et al. [5] and Soref et al. [6], (6) in this paper marks a clear separation between the boundary of single-mode and multimode regions over a large range of etch depth and rib width values, giving us additional confidence in the equation. 


\section{CONCLUSION}

In this paper, the authors have systematically analyzed the design parameters of rib-waveguide structures to satisfy both the single-mode and polarization-independence conditions for small-cross-section SOI waveguides. The requirement of birefringence-free waveguides can be fulfilled by using a deep etch depth when using small-cross-sectional waveguide dimensions; it is more restrictive to design waveguide dimensions to achieve single-mode operation and polarization independence simultaneously. Not surprisingly, the scalar approximation is accurate enough to design single-mode waveguides with large cross section for $r>0.5$, in agreement with results in [5] and [7]. However, for deeply etched small waveguides, the situation is more difficult. The authors have proposed approximate design rules for small waveguides, but for critical designs, individual design simulations should be used to achieve single-mode and birefringence-free operation simultaneously.

\section{ACKNOWLEDGMENT}

The authors would like to thank W. Headley and G. Masanovic for useful discussion. S. P. Chan and S. T. Lim would like to thank Agilent Technologies, Singapore, and the University of Surrey, Surrey, U.K., for partially funding their Ph.D. studies. C. E. Png would like to thank Overseas Research Student (ORS) Award Scheme, Bookham Technology, Inc., and the Institution of Electrical Engineers (IEE) for funding his Ph.D. studies.

\section{REFERENCES}

[1] C. E. Png, S. P. Chan, S. T. Lim, and G. T. Reed, "Optical phase modulators for $\mathrm{MHz}$ and $\mathrm{GHz}$ modulation in silicon-on-insulator (SOI)," $J$. Lightw. Technol., vol. 22, no. 6, pp. 1573-1582, Jun. 2004.

[2] W. R. Headley, G. T. Reed, A. Liu, M. Paniccia, and S. Howe, "Polarization independent optical ring resonator on silicon-on-insulator," Appl. Phys. Lett., vol. 85, pp. 5523-5525, Nov. 2004.

[3] S. P. Chan, V. M. N. Passaro, S. T. Lim, C. E. Png, and G. T. Reed, "Characterization of integrated Bragg gratings on SOI rib waveguides," in Proc. SPIE, vol. 5248, 2003, pp. 273-283.

[4] O. Boyraz and B. Jalali, "Demonstration of a silicon Raman laser," Opt. Express, vol. 12, pp. 5269-5273, Oct. 2004.

[5] R. A. Soref, J. Schmidtchen, and K. Petermann, "Large single-mode rib waveguides in $\mathrm{Ge}-\mathrm{Si}$ and $\mathrm{Si}-$ on-SiO ${ }_{2}$," IEEE J. Quantum Electron., vol. 27, no. 8, pp. 1971-1974, Aug. 1991.

[6] S. P. Pogossian, L. Vescan, and A. Vonsovici, "The single-mode condition for semiconductor rib waveguides with large cross section," $J$. Lightw. Technol., vol. 16, no. 10, pp. 1851-1853, Oct. 1998.

[7] O. Powell, "Single-mode condition for silicon rib waveguides," $J$. Lightw. Technol., vol. 20, no. 10, pp. 1851-1855, Oct. 2002.

[8] L. Vivien, S. Laval, B. Dumont, S. Lardenois, A. Koster, and E. Cassan, "Polarization-independent single-mode rib waveguides on silicon-on-insulator for telecommunication wavelengths," Opt. Commun., vol. 210 , pp. 43-49, Sep. 2002.

[9] J. Lousteau, D. Furniss, A. Seddon, T. M. Benson, A. Vukovic, and P. Sewell, "The single-mode condition for silicon-on-insulator optical rib waveguides with large cross section," J. Lightw. Technol., vol. 22, no. 8, pp. 1923-1929, Aug. 2004.
[10] "Beamprop," Rsoft Design Group, Inc., Ossining, NY.

[11] "FEMLAB," COMSOL, Inc., Burlington, MA.

[12] W. N. Ye, D. X. Xu, S. Janz, P. Cheben, A. Delage, M. J. Picard, B. Lamontagne, and N. G. Tarr, "Stress-induced birefringence in silicon-oninsulator (SOI) waveguides," in Proc. SPIE, vol. 5357, Jul. 2004, pp. $57-66$.

[13] A. G. Rickman, G. T. Reed, and F. Namavar, "Silicon-on-insulator optical rib waveguide loss and mode characteristics," J. Lightw. Technol., vol. 12, no. 10, pp. 1771-1776, Oct. 1994.

[14] A. Sakai, T. Fukazawa, and T. Baba, "Low loss ultra-small branches in a silicon photonics wire waveguide," IEICE Trans. Electron., vol. E85-C, pp. 1033-1038, 2002.

[15] K. K. Lee, D. R. Lim, H. Luan, A. Agarwal, J. Foresi, and L. C. Kimerling, "Effect of size and roughness on light transmission in a $\mathrm{Si} / \mathrm{SiO}_{2}$ waveguide: Experiments and model," Appl. Phy. Lett., vol. 77, pp. 1617-1619, 2000.

[16] J. Wong, J. Well, C. Whiting, C. Yu, B. Porth, J. Hart III, G. Matteson, H. Mochiki, and M. Hagihara, "A hardmask STI etching process for 0.13 $\mu \mathrm{m}$ logic technology and beyond," in IEEE/SEMI Adv. Semiconductor Manufacturing Conf., 2001, pp. 85-94.

[17] International Technology Roadmap for Semiconductors (ITRS). [Online]. Available: http://public.itrs.net/

[18] S. Bosch-Charpenay, X. Jiazhan, J. Haigis, P. A. Rosenthal, P. R. Solomon, and J. M. Bustillo, "Real-time etch-depth measurements of MEMS devices," J. Microelectromech. Syst., vol. 11, pp. 111-117, 2002.

[19] M. Hankinson, T. Vincent, K. B. Irani, and P. P. Khargonekar, "Integrated real-time and run-to-run control of etch depth in reactive ion etching," IEEE Trans. Semicon. Manuf., vol. 10, no. 1, pp. 121-130, Feb. 1997.

Seong Phun Chan was born in Malaysia in 1976. He received the B.Eng. degree (hons.) from the School of Electronics and Physical Sciences, University of Surrey, Surrey, U.K., in 2000 . He is currently working toward the Ph.D. degree at the same university.

His research interests include integrated optics in silicon-on-insulator (SOI) numerical simulation of optical components, waveguide-based Bragg grating devices, and thermooptic phenomena in SOI waveguides.

Mr. Chan was awarded a scholarship by Agilent Technologies, Singapore, to pursue his Ph.D. under the supervision of Prof. G. T. Reed.

Ching Eng Png was born in Singapore in 1974. He received the B.Eng. degree in electronic and electrical engineering (first-class hons.) and the Ph.D. degree from the University of Surrey, Surrey, U.K., in 1999 and 2004, respectively. His $\mathrm{Ph}$.D. work was supervised by Prof. G. T. Reed and focused on silicon photonics.

From November 1999 to September 2000, he was with Agilent Technologies, Singapore, as a Gigabit Optical Transceiver Engineer. He is currently with the Institute of High Performance Computing, A * Star, Singapore, working on biophotonics and silicon photonics.

Dr. Png was awarded the Royal Academy of Engineering Prize and the Institution of Electrical Engineers (IEE) Hudswell International Research Scholarship for his work on silicon optical modulators.

Soon Thor Lim was born in Singapore in 1975. He received the Electrical and Electronic degree (first-class hons.) from the University of Surrey, Surrey, U.K., in 2000. He is currently working toward the Ph.D. degree in silicon photonics with particular emphasis on design and modeling arrayed-waveguide grating (AWG) at the same university.

Mr. Lim was awarded a scholarship from Agilent Technologies, Singapore, to pursue his Ph.D. degree under the supervision of Prof. G.T Reed in 2001. 
Graham T. Reed joined the University of Surrey, Surrey, U.K., in 1989 with the aim of establishing a research activity in guided-wave optoelectronics and now leads an internationally recognized group. He is responsible for initiating a new research field in the United Kingdom on silicon integrated optical circuits, and his group has produced a series of leading technical advances in the field worldwide, notably in optical modulators, grating couplers, and optical sensing applications. A testament to the originality and potential of the silicon work, Bookham Technology plc adopted it as their core business during the early 1990s. His work is built upon collaborative arrangements with both industry and academia alike and has been associated with companies and universities in the United Kingdom, the United States, France, Italy, Germany, Japan, and Singapore. He continues to publish his work and has contributed to more than 80 publications in the field of guided-wave optoelectronics.
Vittorio M. N. Passaro (M'92) was born in Bari, Italy, on November 23, 1962. He received the Laurea degree (cum laude) in electronic engineering and the $\mathrm{Ph} . \mathrm{D}$. degree in electronic engineering, curriculum optoelectronics from the University of Bari, Bari, Italy, in 1988 and 1992, respectively,

Since October 2000, he has been an Associate Professor of electronic technologies at Politecnico di Bari, Bari, Italy. Since 1988, his research interests focused on several theoretical and experimental aspects of optoelectronic technologies and integrated optics. He is involved, often with original contributions, in the modeling, design, and simulation of a number of integrated optical devices and circuits for optical signal processing, telecommunications and sensor applications, by considering a number of materials, in particular ferroelectric semiconductors (silicon and III-V alloy compounds), and ferromagnetic garnets. He has also been involved in dry etching and proton exchange technologies, in particular on the understanding of the physical-chemical mechanisms occurring during the formation of lithium niobate optical waveguides, as well as the relationships with their optical properties. Currently, he is involved in silicon photonics technology. He is author or coauthor of more than 120 papers published in international journals and conference proceedings and two international patents.

Dr. Passaro is a Member of the IEEE Lasers \& Electro-Optics Society (LEOS), the Optical Society of America (OSA), The International Society for Optical Engineers (SPIE), Italian Society of Optics and Photonics (SIOF), the Associated Electrical Industries (AEI), and the National Institute for the Physics of Matter (INFM). 